\title{
LESÃO IATROGÊNICA DE VIAS BILIARES
}

\section{IATROGENIC BILE DUCT INJURIES}

\author{
Guilherme Brasileiro de Aguiar' ${ }^{1}$; Carlos Iglezias Brandão de Oliveira, TCBC-PI'; \\ Joaquim Barbosa de Sousa e Silva Júnior ${ }^{3}$; Loryenne Soares Santos ${ }^{4}$; Sabas Carlos Vieira ${ }^{5}$
}

\begin{abstract}
RESUMO: Objetivo: O objetivo do presente estudo foi avaliar os casos de lesões iatrogênicas de vias biliares tratados na clínica cirúrgica do Hospital Getúlio Vargas - HGV nos últimos oito anos. Método: Foi realizada análise retrospectiva dos prontuários dos pacientes internados no HGV, com hipótese diagnóstica de lesão iatrogênica de vias biliares. Foram incluídos pacientes com lesões provenientes do próprio serviço e de outras instituições. Os prontuários foram revisados e obtidas as seguintes variáveis: procedência do paciente, idade, sexo, data da cirurgia inicial, sintomas, tempo de evolução, valores da bilirrubina, fosfatase alcalina, AST e ALT, TAP, creatinina, além da cirurgia realizada para correção da lesão, complicações, permanência hospitalar e condições do paciente na alta hospitalar. Resultados: Foram confirmados 10 casos de lesão iatrogênica de vias biliares. Todos os paciente eram do sexo feminino, com idades variando entre 18 e 49 anos. Os sinais ou sintomas mais freqüentes foram icterícia, colúria e dor abdominal. Em relação à terapêutica cirúrgica, a hepático-jejunostomia em "Y" de Roux foi o procedimento mais empregado. Conclusões: As lesões iatrogênicas das vias biliares ainda representam um desafio para os cirurgiões e sua ocorrência está mais relacionada a procedimentos realizados em hospitais não especializados, por cirurgiões sem treinamento adequado. A principal medida a ser adotada é a prevenção da lesão. Após sua ocorrência, esta deve ser corrigida em hospitais com equipe médica treinada (Rev. Col. Bras. Cir. 2005; 32(2): 69-73).
\end{abstract}

Descritores: Trato biliar; Colecistectomia; Doença iatrogênica; Procedimentos Cirúrgicos operatórios; Trato bliar/cirurgia.

\section{INTRODUÇÃO}

As lesões iatrogênicas das vias biliares representam um grande desafio em relação às cirurgias das vias biliares, visto que, mesmo com todos os avanços e melhorias técnicas obtidas na atualidade, sua incidência persiste em torno de 0,2 a $2,9 \%^{1,2}$, sendo, a princípio, maior nas videocolecistectomias ${ }^{1-4}$. Embora baixos, estes índices ganham grande importância já que a colecistectomia é uma das cirurgias eletivas mais realizada em hospitais gerais em todo o mundo ${ }^{5,6}$.

Com a adoção da vídeo-laparoscopia como padrão ouro para o tratamento das doenças benignas das vias biliares $^{7,8}$, notou-se um aumento nos índices de lesões iatrogênicas dessas estruturas, em parte explicada pela inexperiência técnica inicial ${ }^{5,8}$.

Outros fatores associados à maior incidência de lesões iatrogênicas das vias biliares são a curva de aprendizado de novos cirurgiões e residentes (embora essa informação seja ainda muito controversa ${ }^{1,8}$ ) e as variações anatômicas do trato biliar. A realização de colangiografia trans-operatória diminui a incidência de lesão iatrogênica, assim como ajuda na sua deteç̧ão precoce ${ }^{8}$.

O prognóstico está diretamente relacionado às condições subjacentes de cada caso, assim como com o tempo decorrido entre a lesão e sua identificação e tratamento, que se constitui basicamente da reconstrução do trajeto biliar, com ou sem anastomoses bilio-digestivas ${ }^{9,10}$.
O objetivo do presente estudo foi avaliar os casos de lesões iatrogênicas de vias biliares tratados na clínica cirúrgica do Hospital Getúlio Vargas nos últimos oito anos.

\section{MÉTODO}

Foi realizada análise retrospectiva dos prontuários de pacientes internados com hipótese diagnóstica de lesão iatrogênica de vias biliares e daqueles com sintomatologia de icterícia obstrutiva após realização de colecistectomia aberta. Foram incluídos pacientes com lesão iatrogênica provenientes do próprio serviço e de outras instituições. Os prontuários foram revisados e obtidas as seguintes variáveis: procedência do paciente, idade, sexo, data da cirurgia inicial, sintomas, tempo de evolução, valores da bilirrubina, fosfatase alcalina, AST e ALT, TAP, creatinina, além do tipo de cirurgia realizada para correção da lesão, presença de complicações, permanência hospitalar e condições do paciente na alta hospitalar.

\section{RESULTADOS}

Foram confirmados 10 casos de lesão iatrogênica de vias biliares. Todos os paciente eram do sexo feminino. A maioria era proveniente do interior do estado do Piaú, com idades variando entre 18 e 49 anos (média de 37,6 anos).

O tempo de evolução, decorrido entre a cirurgia inicial e o aparecimento dos sintomas relativos à injúria

1. Estudante de Medicina da UFPI.

2. Professor de Clínica Cirúrgica da UFPI.

3. Médico formado pela UFPI.

4. Estudante de Medicina da Universidade Estadual do Piauí.

5. Professor Assistente da Clínica Cirúrgica da UFPI.n

Recebido em 22/11/2004

Aceito para publicação em 18/02/2005

Trabalho realizado na Clínica Cirúrgica do Hospital Getúlio Vargas - HGV e Departamento de Clínica Geral da Universidade Federal do Piauí - UFPI Teresina - PI. 
Tabela 1 - Intervalo de aparecimento dos sintomas e presença de icterícia e colúria.

\begin{tabular}{ccc}
\hline Caso no $^{\mathbf{0}}$ & Aparecimento dos sintomas & Icterícia e colúria \\
\hline 01 & $04 \mathrm{dias}$ & $\mathrm{Sim}$ \\
02 & $06 \mathrm{dias}$ & $\mathrm{Sim}$ \\
03 & $07 \mathrm{dias}$ & $\mathrm{Sim}$ \\
04 & $01 \mathrm{dia}$ & $\mathrm{Sim}$ \\
05 & $21 \mathrm{dias}$ & $\mathrm{Sim}$ \\
06 & $03 \mathrm{dias}$ & $\mathrm{Sim}$ \\
07 & $03 \mathrm{dias}$ & Não \\
08 & $07 \mathrm{dias}$ & Não \\
09 & $06 \mathrm{dias}$ & Sim \\
10 & & Não \\
\hline
\end{tabular}

FONTE: Prontuário Médico - SAME/HGV.

iatrogênica variou de um a 21 dias, com um tempo médio de 6,4 dias. Os sinais ou sintomas presentes consistiam de icterícia (oito) colúria (sete), dor abdominal (seis), acolia fecal (quatro), além de distensão abdominal e prurido, cada um ocorrendo em dois pacientes (Tabelas 1 e 2 ).

A Tabela 3 mostra os valores da bilirrubina total e frações, creatinina, TAP, fosfatase alcalina, além da AST e da ALT dos pacientes no momento da internação hospitalar.

Todos os pacientes receberam alta hospitalar sem sintomas, sendo que três pacientes apresentaram como complicação infecção da ferida operatória. A permanência hospitalar variou de oito a 33 dias. A hepático-jejunostomia em "Y" de Roux foi a técnica cirúrgica mais empregada (sete casos), seguida pela anastomose colédoco-duodenal.

\section{DISCUSSÃo}

A colecistectomia representa a cirurgia abdominal eletiva mais freqüentemente realizada no mundo ${ }^{5,6}$, tendo na lesão iatrogênica de vias biliares uma de suas mais temidas complicações ${ }^{2}$. Essa intercorrência sempre acompanhou a realização do procedimento, com lesões iatrogênicas ocorrendo em uma a cada 800 cirurgias abertas realizadas ${ }^{6}$. Com a adoção da vídeo-cirurgia como padrão ouro para o tratamento da doenças benignas biliares ${ }^{\mathbf{1 , 3 , 7 , 8 1 1}}$, houve um incremento na sua incidência, chegando a uma lesão para cada 120 colecistectomias laparoscópicas, segundo alguns autores ${ }^{6}$.
Outros registraram aumento menos expressivo (o dobro em relação às cirurgias abertas ${ }^{3,11}$ ), mas atribuiram maior gravidade à lesão ocorrida durante a vídeo-colecistectomia ${ }^{8}$. Atualmente, não há dúvidas quanto às melhorias introduzidas com o advento da vídeo-cirurgia, porém pôde-se notar um aumento na incidência dos casos de iatrogenia ${ }^{11}$.

O maior índice de injúrias iatrogênicas às vias biliares após vídeo-colecistectomias foi atribuído, no início, à inexperiência do operador ${ }^{1,6,8}$, assim como à "curva de aprendizado" de novos cirurgiões e residentes ${ }^{1,8}$, criando a expectativa de redução desses índices com o ganho de mais experiência $^{8}$. Com a realização cada vez mais freqüente do procedimento, e com a persistência dos índices de iatrogenia, constatouse não se tratar apenas de um processo temporário ${ }^{1,6,8}$, mas de um problema persistente em todo o mundo e não relacionado somente à inexperiência inicial referida. Assim, vários fatores podem ser relacionados ao maior risco de surgimento de lesões iatrogênicas das vias biliares, tanto em cirurgias abertas como em colecistectomia videolaparoscópica ${ }^{3,6}$ : sexo masculino, doença calculosa complicada ${ }^{3,6}$, acesso inadequado, obesidade $^{6,12}$, além da existência de processo inflamatório crônico e sangramento ${ }^{6}$. O risco de lesão iatrogênica também está aumentado nas colecistites agudas em relação as colecistites crônicas $(1,3 \text { vs } 0,6 \%)^{6}$. Outro fator sempre implicado na gênese das lesões de ductos biliares é a variação da anatomia das vias biliares ${ }^{3,6,13}$. Para Sportelli et al ${ }^{13}$ a variação anatômica está presente em 6-25\% dos casos de lesão iatrogênica de acordo com diferentes estatísticas. A anomalia mais comum consiste num ducto hepático direito aberrante presente entre o Triângulo de Calot ${ }^{6}$.

$\mathrm{O}$ pronto reconhecimento da injúria provocada às vias biliares é de suma importância para a melhor recuperação do paciente ${ }^{5,8,10,14}$, porém a presença da lesão provocada só é imediatamente reconhecida em menos da metade dos $\operatorname{casos}^{1,8,10}$. Slater et al $^{\mathbf{8}}$ estudando 131 pacientes com lesão iatrogênica de vias biliares após videocolecistectomia, afirmam que somente $39 \%$ dos casos tiveram seu diagnóstico no intraoperatório. Já Abdel et al ${ }^{10}$ obtiveram a taxa de $10 \%$ das lesões iatrogênicas diagnosticadas durante o ato cirúrgico, seja convencional ou por laparoscopia, sendo que o restante se apresentou em um prazo variável: entre uma semana e dois meses. O mesmo ocorreu em nosso estudo, onde somente

Tabela 2 - Principais sinais e sintomas de cada caso.

\begin{tabular}{|c|c|c|c|c|c|c|c|}
\hline $\begin{array}{l}\text { Sintomas } \\
\text { CASO No }\end{array}$ & Icterícia & $\begin{array}{c}\text { Febre }+ \\
\text { Calafrios } \\
\end{array}$ & $\begin{array}{c}\text { Dor } \\
\text { Abdominal } \\
\end{array}$ & $\begin{array}{c}\text { Distensão } \\
\text { Abdominal }\end{array}$ & Prurido & Colúria & Acolia Fecal \\
\hline 01 & + & - & - & - & - & + & - \\
\hline 02 & + & - & + & - & - & + & + \\
\hline 03 & + & - & - & - & - & + & - \\
\hline 04 & + & - & - & + & - & + & - \\
\hline 05 & + & - & + & - & + & + & + \\
\hline 06 & + & - & - & - & + & + & + \\
\hline 07 & + & - & + & + & - & - & + \\
\hline 08 & - & - & + & - & - & - & - \\
\hline 09 & + & - & + & - & - & + & - \\
\hline 10 & - & - & + & - & - & - & - \\
\hline
\end{tabular}


Tabela 3 - Valores da bilirrubina total e frações, creatinina, TAP, fosfatase alcalina, AST, ALT.

\begin{tabular}{|c|c|c|c|c|c|c|c|c|}
\hline CASO $\mathbf{N}^{\circ}$ & BT (mg/dl) & BD(mg/dl) & BI(mg/dl) & $\mathrm{Cr}(\mathrm{mg} / \mathrm{dl})$ & TAP & FA(KA/dL) & AST(UI/dl) & $\operatorname{ALT}(\mathbf{U I} / \mathbf{d l})$ \\
\hline 01 & 20,4 & 18,7 & 1,7 & 0,54 & $16,1 "$ e $56 \%$ & 377 & 50 & 39 \\
\hline 02 & $\mathrm{NE}$ & $\mathrm{NE}$ & $\mathrm{NE}$ & 1,2 & 15,0 " e $80 \%$ & 129 & 36 & 61 \\
\hline 03 & $\mathrm{NE}$ & $\mathrm{NE}$ & $\mathrm{NE}$ & 0,9 & 12,0 " e $95 \%$ & 164 & 190 & 132 \\
\hline 04 & 14,9 & 11,9 & 3,0 & 1,5 & 13,0 " e $88 \%$ & 115 & $\mathrm{NE}$ & $\mathrm{NE}$ \\
\hline 05 & 13,5 & 11,8 & 1,7 & 0,6 & 14,0 " e $100 \%$ & $\mathrm{NE}$ & 57 & $\mathrm{NE}$ \\
\hline 06 & $\mathrm{NE}$ & $\mathrm{NE}$ & $\mathrm{NE}$ & 3,0 & 17,0 " e $56 \%$ & NE & $\mathrm{NE}$ & $\mathrm{NE}$ \\
\hline 07 & $\mathrm{NE}$ & $\mathrm{NE}$ & $\mathrm{NE}$ & $\mathrm{NE}$ & 18,0 "e $53 \%$ & $\mathrm{NE}$ & $\mathrm{NE}$ & $\mathrm{NE}$ \\
\hline 08 & $\mathrm{NE}$ & $\mathrm{NE}$ & $\mathrm{NE}$ & $\mathrm{NE}$ & $\mathrm{NE}$ & $\mathrm{NE}$ & $\mathrm{NE}$ & $\mathrm{NE}$ \\
\hline 09 & 7,23 & 5,41 & 1,82 & 0,9 & $\mathrm{NE}$ & NE & $\mathrm{NE}$ & $\mathrm{NE}$ \\
\hline 10 & 1,7 & 1,0 & 0,7 & 0,9 & 14,0 " e $100 \%$ & $\mathrm{NE}$ & $\mathrm{NE}$ & $\mathrm{NE}$ \\
\hline
\end{tabular}

LEGENDA: NE: valor não especificada no prontuário.

FONTE: Prontuário Médico - SAME/HGV

uma das dez pacientes avaliadas teve sua lesão identificada no intra-operatório, o que dificultou a classificação e posterior correção cirúrgica da iatrogenia. Nas pacientes estudadas, o tempo de início dos sintomas e conseqüente reconhecimento das lesões existentes variou de um a 21 dias, com uma média de 6,4 dias. Os principais sinais e sintomas referidos foram icterícia, colúria, dor abdominal, acolia fecal, prurido e distensão abdominal, em ordem de freqüência, estando de acordo com o que a literatura aponta.

Quanto às lesões, a literatura não apresenta uma classificação universalmente aceita para agrupá-las ${ }^{15}$, no entanto, a maioria dos autores utiliza em seus trabalhos as classificação de Way $^{16}$, Csendes ${ }^{17}$ (Tabela 4) ou a de Strasberg/ Bismuth $^{16,18-20}$. No presente estudo, as lesões não foram classificadas em decorrência da escassez de dados nos prontuários, impossibilitando, como já mencionado, a determinação da localização e tipo precisos das lesões.

A confirmação diagnóstica é feita através de exames de imagem como a colangiopancreatografia endoscópica retrógrada (CPRE), a colangiografia percutânea trans-hepática, a colangiorressonância (MRCP) ${ }^{13,21}$ e mesmo ultra -sonografia e tomografia computadorizada ${ }^{13}$. No presente trabalho, pôdese confirmar o diagnóstico de iatrogenia através da MRCP (dois pacientes), da CPRE e da US.

No entanto, desde que Mirizzi, em 1931, introduziu a colangiografia intraoperatória ${ }^{22}$, a maior controvérsia em torno do seu uso não está no diagnóstico tardio da lesão, mas sim no valor de sua realização durante o ato cirúrgico para prevenção e possível identificação de lesões do trato biliar ${ }^{8,22,23}$.
Atualmente, recomenda-se sua realização como parte do tempo cirúrgico em procedimentos envolvendo vias biliares na intenção de diminuir ou prevenir possíveis iatrogenias ${ }^{6}$. Alguns autores afirmam que seu uso rotineiro durante o ato cirúrgico reduz o risco e a gravidade da injúria, aumentando as chances de seu pronto reconhecimento e conseqüente reparo cirúrgico, acarretando melhor prognóstico.

Piacentini et $a l^{22}$ avaliaram o uso rotineiro da colangiografia em colecistectomias laparoscópicas e afirmam que sua realização de rotina não diminui a ocorrência de lesão às vias biliares, além de acrescentar, em média, 27 minutos ao tempo cirúrgico e elevar os custos do ato operatório. Afirmou, porém, que ela é efetiva para demonstrar presença de coledocolitíase sem suspeita clínica. Mesmo quando a colangiografia intraoperatória é empregada de rotina, ela apresenta índices de falha que podem chegar a $2 / 3$ dos casos, não revelando a presença da lesão já existente ${ }^{8}$. A recomendação ou não de sua realização ainda é motivo de controvérsia, necessitando de novos estudos para que se obtenha uma posição precisa ${ }^{22}$. Nos casos estudados, não foram realizadas colangiografias no intra-operatório, obtendo-se, o diagnóstico de somente um caso (10\%) durante o ato cirúrgico.

No presente estudo notamos que $90 \%$ dos pacientes (nove em 10) era proveniente de cidades do interior dos estados do Piauí, Ceará, Tocantins e Maranhão, tendo sido submetidos à cirurgia inicial nas respectivas cidades. Representando os pacientes da capital, somente $10 \%$ dos casos, o que demonstra a necessidade de maior experiência e treinamento

Tabela 4 - Classificação das lesões iatrogênicas de Vias Biliares segundo Way e Csendes.

\begin{tabular}{|c|c|c|}
\hline Tipo Classificação & Way & Csendes \\
\hline I & Pequena lesão ou transecção incompleta; & $\begin{array}{l}\text { Pequenas lesões no ducto hepático comum ou direito por } \\
\text { eletrocautério ou tesoura; }\end{array}$ \\
\hline II & Estenose por eletrocautério ou "clip"; & $\begin{array}{l}\text { Lesão causada por tração excessiva do ducto cístico na junção } \\
\text { cístico-hepática; }\end{array}$ \\
\hline III & $\begin{array}{l}\text { Transecção completa com amputação de ducto } \\
\text { hepático comum ou loba; }\end{array}$ & Lesão total ou parcial do ducto colédoco menor que $1 \mathrm{~cm}$; \\
\hline IV & Amputação do ducto hepático direito; & $\begin{array}{l}\text { Lesão do ducto colédoco com ressecção de segmento maior } \\
\text { que } 1 \mathrm{~cm} \text {. }\end{array}$ \\
\hline
\end{tabular}

FONTE: Sawaya DE et al. ${ }^{16}$ e Csendes A et al. 
cirúrgico dos médicos do interior dos estados para que esta grave lesão seja evitada ou pelo menos minimizada.

Em relação ao procedimento cirúrgico, a literatura aponta a hepático-jejunostomia em " $Y$ " de Roux como a melhor e mais freqüentemente empregada opção terapêutica ${ }^{3,9,10,14,20,24-26}$. As lesões do presente estudo foram corrigidas através de hepático-jejunostomia em "Y" de Roux em 70\% dos casos (sete pacientes) e de anastomose colédoco-duodenal nos três casos restantes. A cirurgia reparadora deverá ser precedida por um préoperatório cuidadoso, incluindo um controle de sepse, com drenagem de coleções de bile ou fístulas porventura existentes, além de colangiografia completa ${ }^{3}$. Ainda assim, fatores como a ocorrência de colangite após a lesão biliar, ausência de estudo colangiográfico e realização de cirurgia em menos de três semanas após a injúria, estão associados com maiores chances de falha da reconstrução do trato biliar ${ }^{27}$.

As lesões podem ainda, em casos selecionados, ter sua correção realizada por técnicas de radiologia intervencionista $^{11,14}$, ou mesmo pela simples colocação de dreno em "T" de Kehr, em lesões mínimas identificadas no decorrer da cirurgia ${ }^{7,13}$. Em pacientes sem condições cirúrgicas, a dilatação com balão ou a colocação de stents constituem ações paliativas válidas ${ }^{24}$ enquanto se aguarda um melhor momento cirúrgico. O tratamento conservador, para Sportelli et al ${ }^{13}$ estaria indicado para pacientes portadores de lesões menores, com até $100 \%$ de sucesso, reservando a cirurgia para as lesões maiores.

A prevenção de lesões constitui o principal alvo a ser atingido quando da realização de colecistectomias, seja aberta ou laparoscópica ${ }^{8,24}$ e, caso ocorram, o paciente deverá ser encaminhado a centros com especialistas em cirurgia biliar para tratamento adequado. O prognóstico desses pacientes está diretamente relacionado ao tempo decorrido até a identificação da lesão e seu tratamento ${ }^{5}$, assim como à escolha do procedimento cirúrgico e da habilidade com que é realizado ${ }^{2}$, sendo o estado funcional do fígado o fator isolado mais determinante do prognóstico final².

\begin{abstract}
Background: The objective of this study was to evaluate the cases of iatrogenic lesions of the biliary tract treated in the surgical clinic at Getulio Vargas Hospital - GVH during the last eight years. Methods: A retrospective analysis of the patients' charts admitted with the diagnosis of iatrogenic lesions of the biliary tract was performed. Patients with iatrogenic lesions originating from our service and from other institutions were included. The charts were reviewed for the following variables: origin of the patient, age, sex, date of first surgery, symptoms, time elapsed, bilirubin, alkaline phosphate, AST and ALT, TAP, creatine, surgery performed to correct the lesion, presence of complications, hospital stay and patient status on discharge. Results: Ten cases of iatrogenic lesions of the biliary tract were confirmed. All patients were females, whose ages varied from 18 to 40 . The most frequent signs and symptoms were icterus, chromaturia and abdominal pain. In regards to the corrective surgery, hepatic-jejunostomy in " $Y$ " of Roux was the procedure most frequently used. Conclusion: Iatrogenic lesions of the biliary tract still represent a challenge to general surgeons. It is an occurrence more closely related to procedures performed in nonspecialized hospitals by surgeons without adequate training. The main measure to be taken is to prevent the damage. After the event, it should be corrected by a trained medical team in a well-equipped hospital.
\end{abstract}

Key-words: Biliary tract; Cholecystectomy; Iatrogenic disease; Surgical Procedures, operative; Biliary tract/surgery.

\section{REFERÊNCIAS}

01. Windsor JA, Pong $\mathbf{J}$ - Laparoscopic biliary injury: more than a learning curve problem. Aust N Z J Surg. 1998, 68(3):186-189.

02. Lopéz Smithe O - Lesiones iatrogénicas de la vía biliar. Bol Hosp San Juan de Dios. 1991, 38(5):286-288.

03. Ahrendt SA, Pitt HA - Surgical therapy of iatrogenic lesions of biliary tract. World J Surg. 2001, 25(10):1360-1365.

04. Frühauf NR, Malago M, Broelsch CE - Management of iatrogenic lesions of the biliary tract. Med Sci Monit. 2001, 7 Suppl 1, 1:60-163.

05. Bakhsh R, Zahid MA, Dar FS, et al. - Iatrogenic bile duct injuries: experience at PIMS. J Ayub Med Coll Abbottabad. 2002, 14(4):16-18.

06. Townsend CM Jr., Beauchamp RD, Evers M, et al. (eds) Sabiston Textbook of Surgery: The biological basis of modern surgical practice. $16^{\text {th }}$ Edition. Philadelphia-Saunders Company, 2001.

07. Machado MAC, Rocha JRM, Herman P, et al. - Tratamento laparoscópico de coledocolitíase. Arq Gastroenterol. 2000, 37(3):183-186.
08. Slater K, Strong RW, Wall DR, et al. - Iatrogenic bile duct injury: the scourge of laparoscopic cholecystectomy. ANZ J Surg. 2002, 72(2):83-88.

09. Coelho JCU, Marchesin JB, Souza FJ, et al. - Lesäo iatrogênica das vias biliares. Rev Bras Cir. 1988, 78(5):297-300.

10. Abdel Wahab M, el-Ebiedy G, Sultan A, et al. Postcholecystectomy bile duct injuries: experience with 49 cases managed by different therapeutic modalities. Hepatogastroenterology. 1996, 43 (11):1141-1147.

11. Rossi M, Salvatori FM, Giglio L, et al. - Interventional radiology techniques in the treatment of complications due to videolaparoscopic cholecystectomy. Radiol Med. 2002, 103(4):384-395.

12. Moossa AR, Mayer AD, Stabile B - Iatrogenic injury to the bile duct. Who, how, where? Arch Surg. 1990, 125(8):1028-1030; discussion 1030-1031.

13. Sportelli G, Crovaro M, Mercuri M, et al. - Conservative approach in the treatment of the biliary tract's iatrogenic lesions. Eur Rev Med Pharmacol Sci, 2002, 4(5-6):123-126.

14. Vecchio R, MacFadyen BV, Ricardo AE - Bile duct injury: management options during and after gallbladder surgery. Semin Laparosc Surg. 1998, 5 (2):135-144. 
15. Azagra JS, De Simone P, Goergen M - Is there a place for laparoscopy in management of postcholecystectomy biliary injuries? World J Surg. 2001, 25 (10):1331-1334.

16. Sawaya DE, Johnson LW, Sittig K, et al. - Iatrogenic and noniatrogenic extrahepatic biliary tract injuries: a multiinstitutional review. Am Surg. 2001, 67(5):473-477.

17. Csendes A, Navarrete C, Burdiles P, et al. - Treatment of common bile duct injuries during laparoscopic cholecystectomy: endoscopic and surgical management. Worid J Surg. 2001, 25(10):1346-1351.

18. Strasberg SM, Hertl M, Soper NJ - An analysis of the problem of biliary injury during laparoscopic cholecystectomy. Jour Am Coll Surg. 1995, 180(1):101-125.

19. Murr MM, Gigot JF, Nagorney DM, et al. - Long-term results of biliary reconstruction after laparoscopic bile duct injuries. Arch Surg. 1999,134(6): 604-609.

20. Alvarez GC, Weber ELS, Marcks L, et al. - Lesäo iatrogênica das vias biliares: estudo de 11 casos. J Bras Méd. 1997, 72(1/ 2):76-78.

21. Bujanda L, Calvo MM, Cabriada JL, et al. - MRCP in the diagnosis of iatrogenic bile duct injury. NMR Biomed. 2003, 16(8):475-478.

22. Piacentini F, Perri S, Pietrangeli F, et al. - Colangiografia intraoperatoria in corso di colecistectomia laparoscopica: selettiva o di routine? G Chir. 2003, 24(4):123-128.
23. Woods MS, Traverso LW, Kozarek RA, et al. - Biliary tract complications of laparoscopic cholecystectomy are detected more frequently with routine intraoperative cholangiography. Surg Endosc. 1995, 9(10):1076-1080.

24. Miro AG, De Seta C, Arenga G, et al. - Trattamento delle lesioni iatrogene maggiori della via biliare. Ann Ital Chir. 2002, 73(1):3539.

25. Krawczyk M, Kania M, Zieniewicz K, et al. - [Reconstructive surgery in iatrogenic bile duct injuries]. Wiad Lek. 1997, 50 Suppl 1 Pt 2:313-316.

26. Al-Ghnaniem R, Benjamin IS - Long-term outcome of hepaticojejunostomy with routine access loop formation following iatrogenic bile duct injury. Br J Surg. 2002, 89(9):1118 1124.

27. Chaudhary A, Chandra A, Negi SS, et al. - Reoperative surgery for postcholecystectomy bile duct injuries. Dig Surg. 2002, 19(1):22-27.

Endereço para correspondência:

Guilherme Brasileiro de Aguiar

Rua Osvaldo Costa e Silva, No 1871

Piçarreira

64055-410 - Teresina - PI.

E-mail: guilhermebraguiar@yahoo.com.br 